\title{
APPLICATION OF INTELLIGENT MULTIAGENT APPROACH TO LYME DISEASE SIMULATION
}

\author{
Dmytro Chumachenko1, Tetyana Chumachenko 2 \\ ${ }^{1}$ Informatics Department, National Aerospace University "Kharkiv Aviation Institute", Kharkiv, Ukraine, ${ }^{2}$ Kharkiv National Medical University, Kharkiv, \\ Ukraine
}

\section{Objective}

The objective of this research is to develop the model for calculating the forecast of the Lyme disease dynamics what will help to take effective preventive and control measures using the intelligent multi-agent approach.

\section{Introduction}

Climate warming, globalization, social and economic crises lead to the activation of natural foci of vector-borne infections, among which a special place belongs to Lyme disease (Ixodic tick borreliosis - ITB), the vectors of which are the Ixodes ticks. More than 5,000 cases are registered in the United States every year [1]. In European countries, the number of cases may reach up to 8,00010,000 per year. Incidence rate for ITB in France is 39.4 per 100,000 population, in Bulgaria - 36.6 [2]. In Ukraine, among all ticks, $10-70 \%$ are infected with Borrelia; from $10 \%$ to $42.2 \%$ of Ukrainian population had contact with the causative agent of ITB.

Mathematical modeling as an element of monitoring of natural focal infections makes it possible to assess the epidemiological potential of foci in the region and in individual territories, to forecast the trends of the epidemic process and to determine the main priorities and directions in the prevention of ITB. The most modern and effective method of simulation is multi-agent simulation, which is associated with the concept of an intelligent agent, as some robot, purposefully interacting with other similar elements and the external environment under given conditions. An intelligent agent is an imitation model of an active element, the state and behavior of which in various situations of achieving the goal vary depending on the state and behavior of other agents and the environment, in analogy with the intellectual behavior of a live organism (including a human) under similar conditions.

As the epidemic process of Lyme disease is characterized by vector transmission, heterogeneous tick population, variable pathogen infectivity, heterogeneous environment, and seasonal changes in tick activity, the use of classical statistical methods for predicting the dynamics of morbidity cannot show high accuracy. The multiagent approach to simulation of the epidemic process of Lyme disease allows considering all of the above features, and since the dynamics of the modeled system is formed from the behavior of local objects (humans and ticks), we expect that a model constructed using a multiagent approach will yield a higher accuracy of prognosis morbidity. The multiagent model will allow not only to calculate the forecast, but also to reveal the factors influencing increase of the incidence of Lyme disease the most.

\section{Methods}

The research is based on official reports of new Lyme disease cases registered during 2006-2017. The data were collected by the State Institution Kharkiv Laboratory Center of the Ministry of Health of Ukraine from the healthcare facilities of Kharkiv Oblast of Ukraine as a part of passive routine disease surveillance. In total, 1016 cases of Lyme disease registered during the study period were included in this research.

The multi-agent approach to simulation of epidemic process has been used for the model development. The epidemiological model of Lyme disease is based on the Gromashevsky's concept of the epidemic process, according to which the epidemic process exists with the continuous interaction of the three main components - source of infection, mechanism of transmission and susceptible organism.

The most profitable type of agent in the study of epidemic process is an emotionally-motivated intellectual agent for the most complete and accurate model of human behavior. Let's consider the agent as a set of properties:

$\mathrm{a}=<\mathrm{s}, \mathrm{st}, \mathrm{c}, \mathrm{ta}, \mathrm{l}>, \mathrm{a} \in \mathrm{A}, \mathrm{s} \in \mathrm{S}, \mathrm{c} \in \mathrm{C}, \mathrm{ta} \in \mathrm{Ta},(1)$

SDS Annual Conference Proceedings 2019. This is an Open Access article distributed under the terms of the Creative Commons AttributionNoncommercial 4.0 Unported License (http://creativecommons.org/licenses/by-nc/3.0/), permitting all non-commercial use, distribution, and reproduction in any medium, provided the original work is properly cited. 
where $s t$ is time in state $s, A$ is set of all agents, $S$ is set of different agent's states, $C$ is set of working area's cells, $T a$ is set of possible agent's types, $l$ is length of life. The set of agent states is predefined and is constant. Depending on the process being studied, the set can be supplemented by different states, the initial set is:

$S=\{$ Susceptible, Exposed, Infected, Dead $\} .(2)$

The composition of the workspace leads to the appearance of a set of cells, as conditional abstract objects. It is assumed that one cell can simultaneously include a number of agents as well as one object-vector of infection (tick). It is assumed that depending on the type of working area where the agent is located the specific nature of the epidemic process is changed.

The vector transmission of the disease from tick to agent is realized as follows. To begin with, the possibility of contact with tick is checked. It is believed that this is possible if other agents are also processed in the same cell along with the tick processed as well. Each pair agent-tick is compared. If tick is infected, it is believed that with a certain probability healthy agent can become infected.

To automate the prediction of the incidence of Lyme disease, a software package has been developed using C\# programing language that allows to calculate prognosis morbidity based on existing statistical data in real time. In the developed model, the configuration of the software package includes data for the period 2006-2017. This data include incidence rate of ITB per 100,000 population, the population's seeking healthcare providers for tick bites, the results of studies of ticks removed from humans for the presence of Borrelia, the number and proportion of ticks infected by Borrelia collected by the flagging method.

\section{Results}

The calculated prognosis shows the expected increase in the number of cases with a certain three-year cycle: an increase in the number of cases of Ixodic tick borreliosis within 2 years with a slight decrease for the next year.

A comparative analysis of the accuracy of forecasting by moving average method to 3 years [3] and multiagent simulation showed that the latter describes the existing data better, therefore, the forecast will be more accurate. The accuracy rates using the moving average method is $85.4 \%$, with the use of multiagent simulation $96.6 \%$.

\section{Conclusions}

A comparative assessment of the accuracy of predicting the dynamics of the epidemic process using the moving average method [3] and the multiagent approach was carried out. A higher accuracy is noted with the use of multi-agent simulation (96.6\% against $85.4 \%$ using moving average method), based on which a software package has been developed, which makes it possible to calculate the expected morbidity rate of Lyme disease. That means that the hypothesis made of the research was confirmed. The adequacy of the tested prognosis was verified on the real statistical data collected by the State Institution Kharkiv Laboratory Center of the Ministry of Health of Ukraine on the incidence of Lyme disease in Kharkiv Oblast (Ukraine) from 2006 to 2017 . The obtained forecast revealed the expected persistence of the unstable epidemic situation with respect to Lyme disease, which dictates the need to develop a set of preventive measures aimed at reducing the morbidity of people by ITB. Virtual verification of the effectiveness of such events will be the next step in our study.

\section{References}

1. Gulia-Nuss M, Nuss AB, et al. 2016. Genomic insights into the Ixodes scapularis tick vector of Lyme disease. Nat Commun. 7, 10507. PubMed https://doi.org/10.1038/ncomms10507

2. Lindgren E, Jaenson TGT. Lyme borreliosis in Europe: influences of climate and climate change, epidemiology, ecology and adaptation measures. World Health Organization, 2016. 35 p.

3. Chumachenko T, Chumachenko D, Sukhorukova M. Simulation of the epidemic process of Ixodes tick borreliosis. CBEP Ukraine Regional One Health Research Symposium and Peer Review Session. Kyiv: Cooperative Biological Engagement Program (CBEP), 2017. pp. 168. 